\title{
Microextração Líquido-Líquido Dispersiva (DLLME): fundamentos e aplicações
}

\author{
Manoel Leonardo Martins', Ednei Gilberto Primel2', Sergiane Souza Caldas², \\ Osmar Damian Prestes ${ }^{3}$, Martha Bohrer Adaime', Renato Zanella ${ }^{*}$ \\ ${ }^{1}$ Departamento de Química, Universidade Federal de Santa Maria - UFSM, \\ Cep 97105-900, Santa Maria, RS, Brasil \\ e-mail: rzanella@base.ufsm.br \\ ${ }^{2}$ Escola de Química e Alimentos, Universidade Federal do Rio Grande - FURG, \\ Cep 96201-900, Rio Grande, RS, Brasil \\ ${ }^{3}$ Universidade Federal do Pampa - UNIPAMPA, Campus Itaqui, \\ Cep 97650-000, Itaqui, RS, Brasil
}

\section{Resumo}

Nos últimos anos, as técnicas clássicas de preparo de amostra para a determinação de compostos orgânicos em amostras ambientais e alimentos estão sendo substituídas por técnicas miniaturizadas que são caracterizadas pelo baixo custo, alta eficiência, reduzida quantidade de solvente, além da possibilidade de automação. Dentre essas técnicas, a Microextraçao Líquido-Líquido Dispersiva (DLLME) apresenta-se como uma interessante alternativa para o preparo de amostra e está baseada na partição dos analitos de interesse empregando-se pequenos volumes de uma mistura de solventes (dispersor e extrator). A DLLME apresenta como principais vantagens a miniaturização, baixo custo, rapidez, alta eficiência de extração e pré-concentração, bem como potencial para aplicação direta em campo. Esta revisão apresenta fundamentos e aplicações da DLLME na determinação de compostos orgânicos em diferentes tipos de amostras.

Palavras-chave

Preparo de amostra; extração miniaturizada; compostos orgânicos; cromatografia.

\section{Dispersive Liquid-Liquid Microextraction (DLLME): fundamentals and applications}

\section{Abstract}

In the last years, the classical sample preparation techniques for the determination of organic compounds in environmental and food samples are being replaced by miniaturized techniques that are characterized by low cost, high efficiency, low solvents consume, with the possibility of automation. Among these techniques, the Dispersive Liquid-Liquid Microextraction (DLLME) presents an interesting alternative for sample preparation and is based in the partition of analytes using a mixture of solvents (disperser and extractor). The DLLME presents as main advantages the miniaturization, low cost, the speed, the efficiency of extraction and preconcentration, as well as the potential for direct application in the field. This review presents the fundamentals and recent applications of DLLME in the analysis of organic compounds in different samples.

Keywords

Sample preparation; miniaturized extraction; organic compounds; chromatography. 


\section{Introdução}

O preparo de amostra é uma das etapas críticas do processo analítico e tem sido um dos principais obstáculos para a obtenção de resultados adequados em termos de precisão e detectabilidade. Para minimizar estes problemas, diferentes métodos têm sido propostos a fim de otimizar e propor alternativas à extração líquido-líquido (LLE, do inglês Liquid-Liquid Extraction) convencional. A LLE é um método clássico de extração e pré-concentração de analitos, ainda muito utilizado. No entanto, caracteriza-se pela demora, requer grandes volumes de solventes orgânicos, além de envolver várias etapas que estão associadas com as perdas de analito e ocorrência de contaminação ${ }^{[1]}$. Atualmente, atenção especial é dispensada para métodos que sejam rápidos, eficientes e ambientalmente corretos. Desta forma, métodos baseados em microextração vêm sendo desenvolvidos como alternativas aos métodos clássicos de extração.

A Microextração Líquido-Líquido Dispersiva (DLLME, do inglês Dispersive Liquid-Liquid Microextraction), proposta em 2006 por REZAEE e colaboradores ${ }^{[2]}$, utiliza um solvente dispersor, miscível no solvente extrator (fase orgânica) e na amostra (fase aquosa), bem como um solvente extrator, imiscível na fase aquosa, sendo baseada em um sistema ternário de solventes como a Extração Líquido-Líquido Homogênea (HLLE, do inglês Homogeneus Liquid-Liquid Extraction) e a Extração em Ponto de Nuvem (CPE, do inglês Cloud Point Extraction) ${ }^{[3]}$. A DLLME é uma técnica de extração e pré-concentração de amostras que atende aos requisitos de miniaturização, baixo custo, rapidez e eficiência de extração e com alto potencial para aplicação direta em campo ${ }^{[4]}$.

Na DLLME, o tempo de extração é definido como o intervalo entre a injeção da mistura de solventes e a centrifugação. A área superficial entre o solvente de extração e a fase aquosa é infinitamente grande. Assim, a transferência dos analitos da fase aquosa para a fase extratora é rápida e, portanto, o estado de equilíbrio é atingido rapidamente ${ }^{[3]}$.

A mistura de solventes é introduzida na amostra em um tubo de fundo cônico, conforme Figura 1, onde ocorre a extração dos analitos por partição e, após, a mistura é centrifugada. O solvente extrator sedimentado no fundo do tubo é recolhido e analisado. $\mathrm{O}$ processo de extração ocorre de forma muito parecida com aquele da Extração Líquido-Líquido tradicional.

Na LLE, a mistura do solvente orgânico com a amostra aquosa é promovida por agitação, e a formação de bolhas ou gotas de solvente orgânico garante o aumento da superfície de troca para favorecer a partição dos analitos entre as fases ${ }^{[3]}$.

Na DLLME, a mistura obtida pela injeção rápida de um jato de uma mistura de solventes, onde um solvente miscível na fase aquosa (solvente dispersor) e na fase orgânica (solvente extrator) promove a dispersão desta última na forma de microgotas com grande área superficial onde ocorre a partição, como está representado na Figura 1.

Outra diferença entre a técnica LLE e a DLLME é que na LLE geralmente são realizadas etapas sucessivas de extração, para aumento da eficiência, enquanto que na DLLME a extração ocorre em uma única etapa. Se a eficiência de extração for próxima a $100 \%$, a técnica DLLME pode ser considerada um técnica exaustiva, caso contrário é considerada uma técnica de equilíbrio ${ }^{[6]}$.

\section{Princípios da DLLME}

A técnica DLLME é normalmente realizada em duas etapas conforme representado na Figura 2. A primeira etapa consiste na injeção de uma mistura adequada dos solventes extrator e 


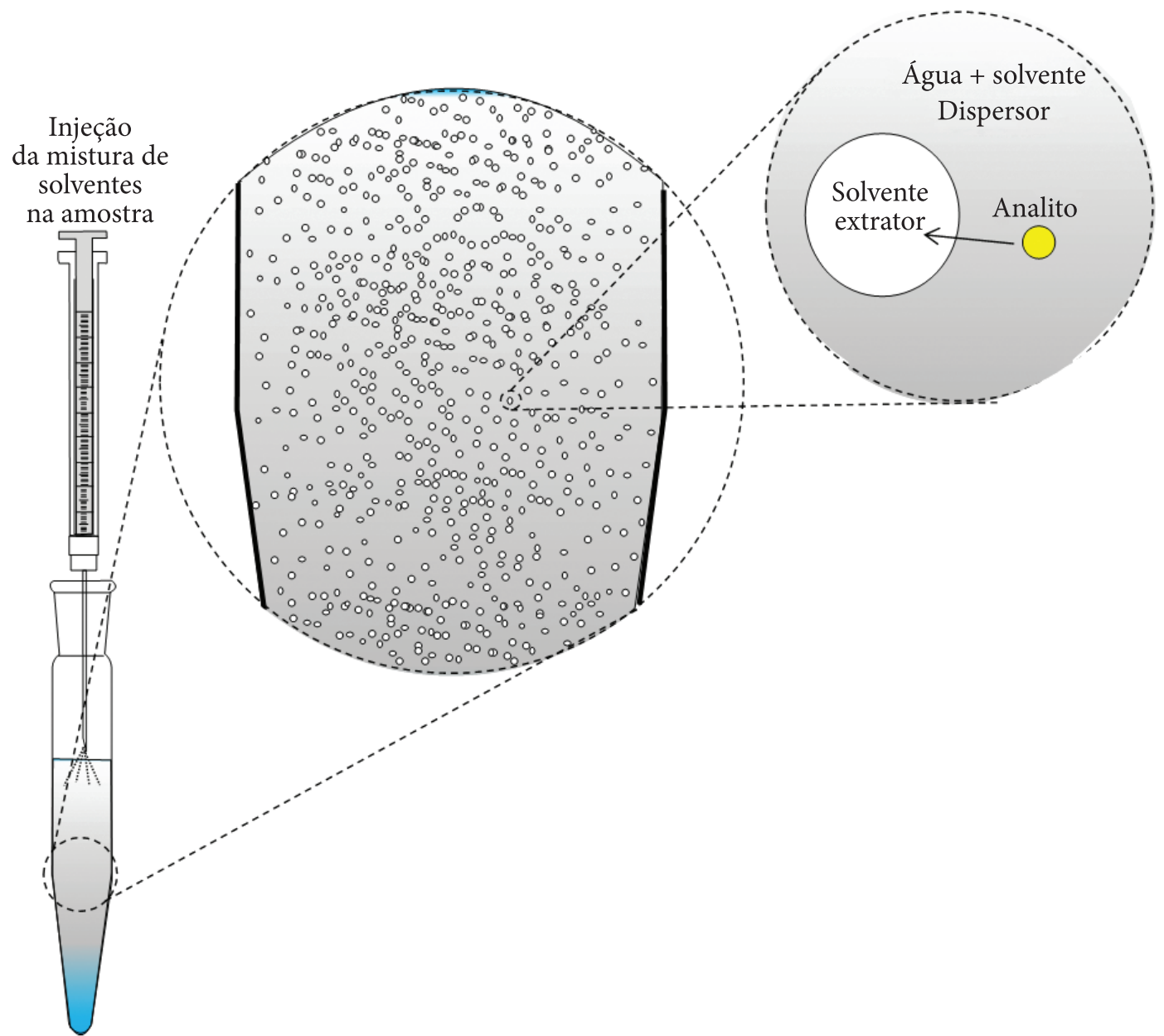

Figura 1 Diagrama simplificado demonstrando a injeção da mistura dos solventes na amostra, a dispersão do solvente extrator na amostra e a partição do analito entre a amostra e o solvente extrator.

dispersor na amostra aquosa contendo os analitos. Nesta etapa, o solvente extrator é disperso na fase aquosa em gotas muito finas extraindo os analitos. Esta dispersão do solvente extrator é favorecida pelo solvente dispersor, que deve ser solúvel na amostra aquosa e na fase orgânica. Devido à grande área superficial entre o solvente extrator e a amostra aquosa, o equilíbrio é atingido rapidamente e a extração é independente do tempo, sendo esta a principal vantagem deste método ${ }^{[3]}$. A segunda etapa é a centrifugação da solução turva e a transferência da fase sedimentada para um frasco (microvial) que permitirá a determinação dos analitos.
A DLLME baseia-se no processo de partição dos analitos entre duas fases líquidas imiscíveis, sendo uma delas a fase aquosa (a amostra) e a outra um fase orgânica (solvente orgânico) A solubilização dos analitos em qualquer uma das fases é governada pela polaridade relativa do sistema, sendo que os analitos são geralmente apolares, apresentando maior afinidade pela fase orgânica, composta por um solvente orgânico apolar, em comparação com a fase aquosa, que é, por sua vez, altamente polar ${ }^{[7]}$.

A polaridade de uma molécula refere-se às concentrações de cargas da nuvem eletrônica em volta da molécula. Moléculas polares possuem 


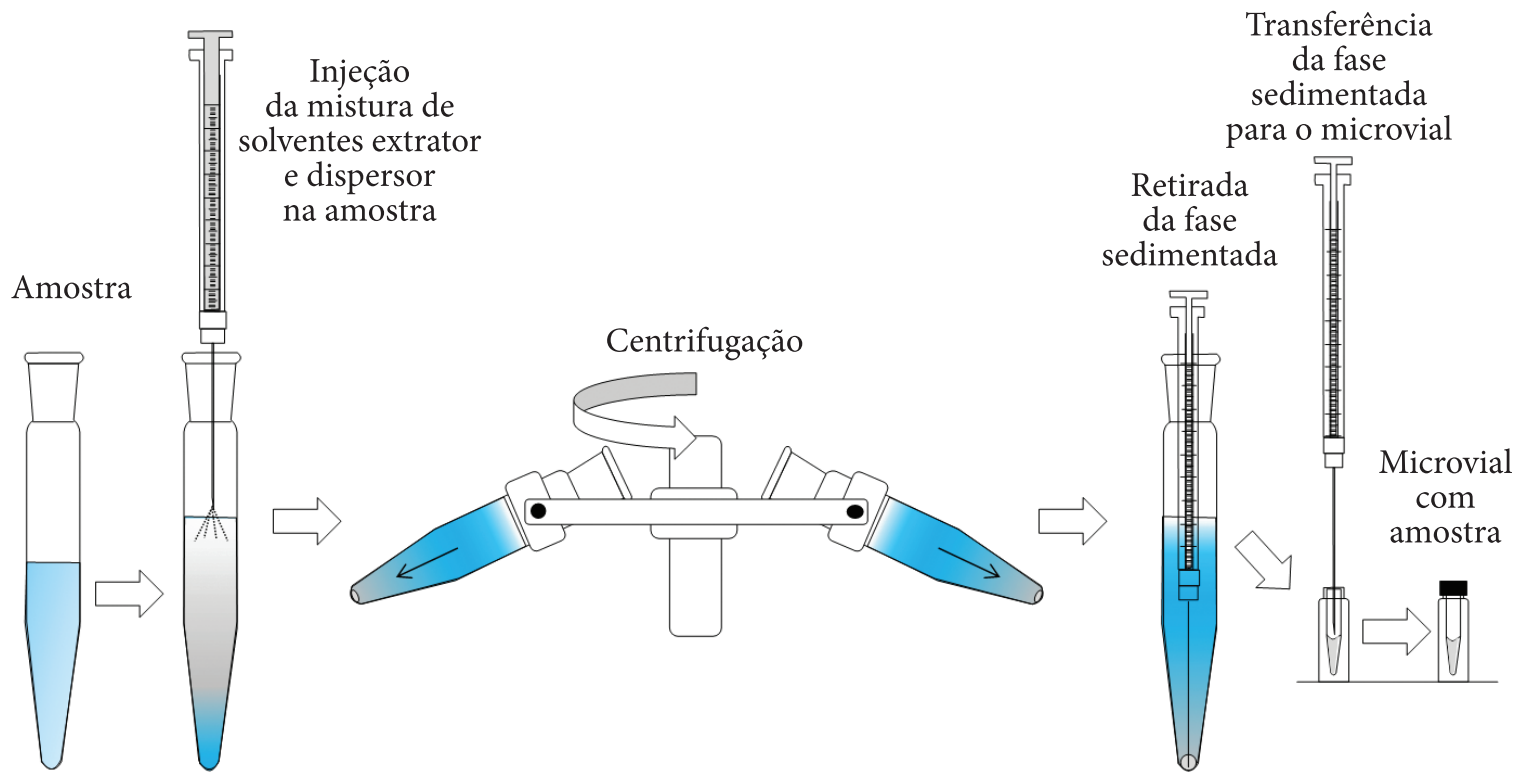

Figura 2 Diagrama simplificado demonstrando as etapas da DLLME, adaptado de Berijani et al. ${ }^{[5]}$

maior concentração de carga negativa numa parte da nuvem e maior concentração positiva em outro extremo. Nas moléculas apolares, a carga eletrônica está uniformemente distribuída. As diferenças de cargas elétricas entre as moléculas dos analitos, da fase aquosa (polar) e da fase orgânica (apolar), é que determinam o equilíbrio resultante ${ }^{[7]}$, que pode ser representado por:

$$
A_{a q} \rightleftharpoons A_{\text {org }}
$$

No caso ideal, a razão das atividades do analito A nas duas fases é uma constante e independe da quantidade de $\mathrm{A}$, sendo que para uma dada temperatura, a constante de equilíbrio $\left(K_{D}\right)$ é o coeficiente de partição ou distribuição ${ }^{[7]}$, sendo representado como:

$$
K_{D}=\frac{\left[A_{o r g}\right]}{\left[A_{a q}\right]}
$$

onde: A: Analito; aq: fase aquosa; org: fase orgânica; $K_{D}$ : coeficiente de distribuição ou partição; [ ]: atividade ou concentração relativa do analito.

Depois do tempo necessário para que seja atingido o equilíbrio da partição do analito, as fases são separadas. Alguns fatores afetam a polaridade relativa do sistema, tais como presença ou adição de sais, alterações no pH da fase aquosa, modificadores químicos ou misturas de solventes solúveis ou parcialmente solúveis na fase aquosa ${ }^{[2]}$.

A fase orgânica ou extrato poderá conter a maior proporção do analito. Se esta quantidade for próxima a $100 \%$ pode ser considerada uma técnica de extração exaustiva e a exatidão do método, expressa em termos de recuperação, pode ser calculada através da seguinte Equação 3:

$$
R(\%)=\frac{C_{1}-C_{2}}{C 3} \times 100
$$

onde: $\mathrm{C}_{1}=$ concentração do analito determinada na amostra fortificada; $C_{2}=$ concentração do analito na amostra não fortificada; $\mathrm{C}_{3}=$ concentração do analito adicionada na amostra fortificada.

Se a transferência do analito entre a fase aquosa e a fase orgânica for parcial, a extração por DLLME será considerada uma técnica de equilíbrio $^{[6]}$. Neste caso, a recuperação $(R)$ pode ser definida como a quantidade total de analito, 
em porcentagem, que é transferida para a fase aceptora (ou orgânica) ao final da extração. A razão entre o volume da fase doadora (aquosa) e a fase aceptora (orgânica) também governa a recuperação do analito, de acordo com a Equação $4^{[8]}$.

$$
R(\%)=\frac{K_{D} V_{\text {org }}}{K_{D} V_{\text {org }}+V_{d}} \times 100
$$

onde: $\mathrm{K}_{\mathrm{D}}$ : coeficiente de distribuição ou partição; $\mathrm{V}_{\text {org }}=$ volume da fase orgânica; $\mathrm{V}_{\mathrm{d}}=$ volume da fase doadora (ou aquosa).

Portanto a extração em duas fases é favorecida para analitos hidrofóbicos, ou seja, com elevados coeficientes de partição (entre 500 e 1000 ou maiores $)^{[8]}$. Adicionalmente, a recuperação pode ser favorecida aumentando-se a razão entre as fases aceptora (orgânica) e doadora ${ }^{[6]}$.

Outro parâmetro comumente utilizado para demonstrar a eficiência dos processos de microextração em fase líquida, como no caso da DLLME, é o fator de enriquecimento (E), que informa o grau de concentração do analito obtido na extração e pode ser calculado pela Equação 5:

$$
E=\frac{V_{d} R}{100 V_{\text {org }}}
$$

como os valores de $\mathrm{V}_{\mathrm{d}} / \mathrm{V}_{\text {org }}$ são normalmente elevados, altos fatores de enriquecimento são obtidos ${ }^{[6,9]}$.

\section{Parâmetros relacionados à eficiência de extração por DLLME}

Os principais fatores que afetam a eficiência da extração por DLLME, são: tipo e volume dos solventes extrator e dispersor. Em DLLME, os fatores mais importantes que afetam o volume da fase sedimentada são: solubilidade do solvente extrator em água, volume e características da amostra, volume do solvente dispersor e volume do solvente extrator. Do ponto de vista experi- mental, para obter um volume adequado de fase sedimentada, alguns testes devem ser efetuados antes do início da otimização ${ }^{[10,11]}$.

\subsection{Seleção do solvente extrator}

O principal parâmetro a ser definido para a otimização de um procedimento de extração por DLLME é o tipo de solvente extrator, que deve ser escolhido com base na sua densidade, capacidade de extração dos analitos de interesse e adequação à técnica analítica escolhida ${ }^{[12]}$.

$\mathrm{Na}$ Tabela 1, estão listadas as propriedades de solventes de uso comum em laboratórios de análises que podem ser utilizados na técnica DLLME como solvente extrator ou como solvente dispersor e algumas de suas propriedades físico-químicas.

O solvente extrator deve ter densidade maior que a água, de forma a permitir a formação da fase sedimentada ${ }^{[14]}$, embora aplicações descrevam a utilização de solventes extratores com densidade menor que a da água ${ }^{[15,16]}$. Tanto o solvente extrator quanto o solvente dispersor devem ter pressão de vapor relativamente baixa e temperatura de ebulição relativamente alta, para evitar perdas significativas de solvente durante o processo de extração ${ }^{[17]}$. Outra característica importante do solvente extrator é ter baixa solubilidade em água, permitindo a separação adequada do extrato orgânico ${ }^{[18]}$.

Quando utilizada a determinação por Espectrometria de Massas, as massas moleculares dos solventes utilizados devem ser consideradas para evitar interferências espectrais. $O$ ponto de fusão do solvente extrator pode ser importante, no caso de aplicação de resfriamento para congelamento da fase sedimentada (gota) para posterior separação da fase aquosa ${ }^{[15]}$. Se for utilizada a Cromatografia Gasosa para separação dos analitos, o ponto de ebulição do solvente extrator será determinante na transferência do analito para a coluna cromatográfica e separação ${ }^{[3]}$. 
Tabela 1 Algumas propriedades físico-químicas de alguns solventes passíveis de serem utilizados como solventes extratores e dispersores.

\begin{tabular}{|c|c|c|c|c|c|c|}
\hline Solvente & $\begin{array}{c}\text { Massa molar } \\
\left(\mathrm{g} \cdot \mathrm{mol}^{-1}\right)\end{array}$ & $\begin{array}{c}\text { Solubilidade } \\
\text { em água } \\
\left(\mathrm{g} \cdot \mathrm{L}^{-1}, 20^{\circ} \mathrm{C}\right)\end{array}$ & $\begin{array}{l}\text { Ponto de } \\
\text { fusão } \\
\left({ }^{\circ} \mathrm{C}\right)\end{array}$ & $\begin{array}{c}\text { Densidade } \\
\left(\mathrm{g} . \mathrm{cm}^{-3}, 20^{\circ} \mathrm{C}\right)\end{array}$ & $\begin{array}{c}\text { Ponto de } \\
\text { ebulição } \\
\left({ }^{\circ} \mathrm{C}, 1013 \mathrm{hPa}\right)\end{array}$ & $\begin{array}{c}\text { Pressão de } \\
\text { vapor } \\
\left(\mathrm{mmHg}, 20^{\circ} \mathrm{C}\right)\end{array}$ \\
\hline \multicolumn{7}{|l|}{ Extratores } \\
\hline $\begin{array}{l}\text { Tetracloreto de } \\
\text { Carbono }\end{array}$ & 153,8 & 0,8 & -23 & 1,59 & 76,7 & 90 \\
\hline Clorofórmio & 119,4 & 8 & -63 & 1,47 & 61 & 158 \\
\hline Diclorometano & 84,9 & 20 & -95 & 1,33 & 40 & 356 \\
\hline 1,2-Dicloroetano & 99,0 & 8,7 & $-35,5$ & 1,25 & $83,5-84,1$ & 65 \\
\hline Acetato de etila & 88,1 & 85,3 & -83 & 0,90 & 77 & 73 \\
\hline 1-Clorobutano & 92,6 & 0,5 & -123 & 0,886 & 79 & 83 \\
\hline Benzeno & 78,1 & 1,8 & 5,5 & 0,88 & 80,1 & 76 \\
\hline Tolveno & 92,1 & 0,52 & -95 & 0,87 & 110,6 & 22 \\
\hline 1-Butanol & 74,1 & 77 & -89 & 0,81 & $116-118$ & 5 \\
\hline $\begin{array}{l}\text { Éter tert- } \\
\text { butil-metílico }\end{array}$ & 88,2 & 42 & $-108,6$ & 0,74 & 55,3 & 201 \\
\hline Ciclo-hexano & 84,2 & 0,055 & 6 & 0,78 & 81 & 77 \\
\hline Iso-octano & 114,2 & 0,00056 & -107 & 0,69 & 99 & 38 \\
\hline n-Heptano & 100,2 & 0,05 & $-90,5$ & 0,68 & $97-98$ & 36 \\
\hline n-Hexano & 86,2 & 0,0095 & $-94,3$ & 0,66 & 69 & 120 \\
\hline \multicolumn{7}{|l|}{ Dispersores } \\
\hline $\begin{array}{l}\text { Tetra- } \\
\text { hidrofurano }\end{array}$ & 72,1 & solúvel & $-108,5$ & 0,89 & $65-66$ & - \\
\hline 1,4-Dioxano & 88,1 & solúvel & 12 & 1,03 & 101,5 & 31 \\
\hline 1-Propanol & 60,1 & solúvel & -127 & 0,80 & $96,5-98$ & 14 \\
\hline Metanol & 32,0 & solúvel & -98 & 0,792 & 64,5 & 96 \\
\hline Etanol & 46,1 & solúvel & $-114,5$ & 0,79 & 78,3 & 44 \\
\hline Acetona & 58,1 & solúvel & $-95,4$ & 0,79 & 56,2 & 175 \\
\hline Acetonitrila & 41,5 & solúvel & $-45,7$ & 0,786 & 81,6 & 73 \\
\hline 2-Propanol & 60,1 & solúvel & $-89,5$ & 0,786 & 82,4 & 32 \\
\hline
\end{tabular}

Fonte: Merck (2010) (http://www.merck-chemicals.com.br/solventes-para-cromatografia-analitica-lichrosolv-)[13].

\subsection{Seleção do solvente dispersor}

O parâmetro fundamental para a escolha do tipo de solvente dispersor é sua solubilidade tanto na fase orgânica quanto na fase aquosa. $\mathrm{O}$ volume de solvente dispersor afeta diretamente a formação das microgotas de água/solvente dispersor/solvente extrator, o grau de dispersão do solvente na fase aquosa e, consequentemente, a eficiência da extração ${ }^{[14]}$.

\subsection{Efeito do volume dos solventes extrator e dispersor}

O volume do solvente extrator determina o fator de pré-concentração da DLLME. Aumentando o volume do solvente extrator, o volume da fase sedimentada obtido com a centrifugação aumenta, resultando em um menor fator de pré-concentração. Portanto, o volume 
ótimo de solvente extrator deve garantir tanto um alto fator de pré-concentração quanto permitir a obtenção de um volume de fase sedimentada suficiente para as análises necessárias ${ }^{[19]}$.

Variações no volume do solvente dispersor causam variações no volume da fase sedimentada, tornando necessário modificar simultaneamente o volume do solvente extrator e o volume do solvente dispersor para manter constante o volume da fase sedimentada. $\mathrm{O}$ volume adequado de solvente dispersor para uma boa formação das microgotas depende tanto do volume da fase aquosa quanto do volume do solvente extrator $^{[18]}$.

\subsection{Efeito do tempo de extração}

Na DLLME, apesar do equilíbrio de partição dos analitos entre a fase orgânica e a fase aquosa ser atingido muito rapidamente, na prática, o tempo de extração é definido como o intervalo entre a injeção da mistura dos solventes extrator e dispersor na fase aquosa e o final da centrifugação. Este também deve ser otimizado e é um dos parâmetros mais importantes em técnicas de microextração ${ }^{[3,20,21]}$.

\subsection{Efeito da adição de sais e do ajuste do $\mathrm{pH}$}

A influência da força iônica também deve ser considerada, uma vez que influencia na separação das fases. O aumento na concentração de $\mathrm{NaCl}$ aumenta o volume de fase sedimentada, devido à diminuição da solubilidade do solvente extrator na presença de um sal. Esta influência será de acordo com as características de cada analito. Observa-se, nos trabalhos publicados, uma grande variação da adição de $\mathrm{NaCl}$, desde a não utilização até a adição de 0,5 a 30\%. Outro parâmetro que deve ser levado em consideração é o $\mathrm{pH}$, que é otimizado em função da acidez ou basicidade de alguns compostos. $\mathrm{O}$ ajuste do
$\mathrm{pH}$ do meio permite que os analitos permaneçam na forma neutra, facilitando a partição dos analitos nas microgotas do solvente extrator ${ }^{[21]}$. No trabalho desenvolvido por Farhadi et al. ${ }^{[22]}$, o pH da amostra foi ajustado em 1,5 para extração de herbicidas da classe dos clorofenoxiacéticos em amostras de água. A amostra com pH 1,5 e $10 \% \mathrm{NaCl}$ foi extraída com $1 \mathrm{~mL}$ acetona (dispersor) e $25 \mu \mathrm{L}$ de clorobenzeno (extrator). Recuperações entre 94 e 102,9\% foram obtidas em águas de poço e de torneira, com limite de detecção (LOD) de 0,16 $\mu \mathrm{g} \cdot \mathrm{L}^{-1}$.

\section{Aplicações}

A DLLME tem sido aplicada com sucesso nas etapas de extração e pré-concentração de uma grande variedade de compostos orgânicos e íons metálicos. A Tabela 2 apresenta as características de algumas aplicações da DLLME para amostras ambientais. Entre os analitos avaliados nestas aplicações pode-se destacar agrotóxicos, fármacos e outros poluentes orgânicos. Como pode ser observado, a DLLME é uma técnica de preparo de amostra bastante versátil, uma vez que os extratos obtidos podem ser empregados com sucesso em distintos métodos de análise.

A determinação de compostos orgânicos em alimentos é bastante complexa devido à presença de coextrativos que podem afetar o resultado final. Entre os problemas que podem ser observados estão: baixa robustez do método, diminuição da detectabilidade e quantificação imprecisa, uma vez que resultados falsos positivos ou falsos negativos podem ser obtidos. Desta maneira, atenção especial vem sendo dada a métodos de preparo de amostra que minimizem o efeito destes coextrativos. Como pode ser observado na Tabela 3, a técnica DLLME tem sido aplicada com sucesso na análise de diferentes compostos orgânicos em alimentos.

$\mathrm{Na}$ grande maioria das aplicações apresentadas nas Tabelas 2 e 3, os solventes de extração empregados são clorados. O emprego destes sol- 


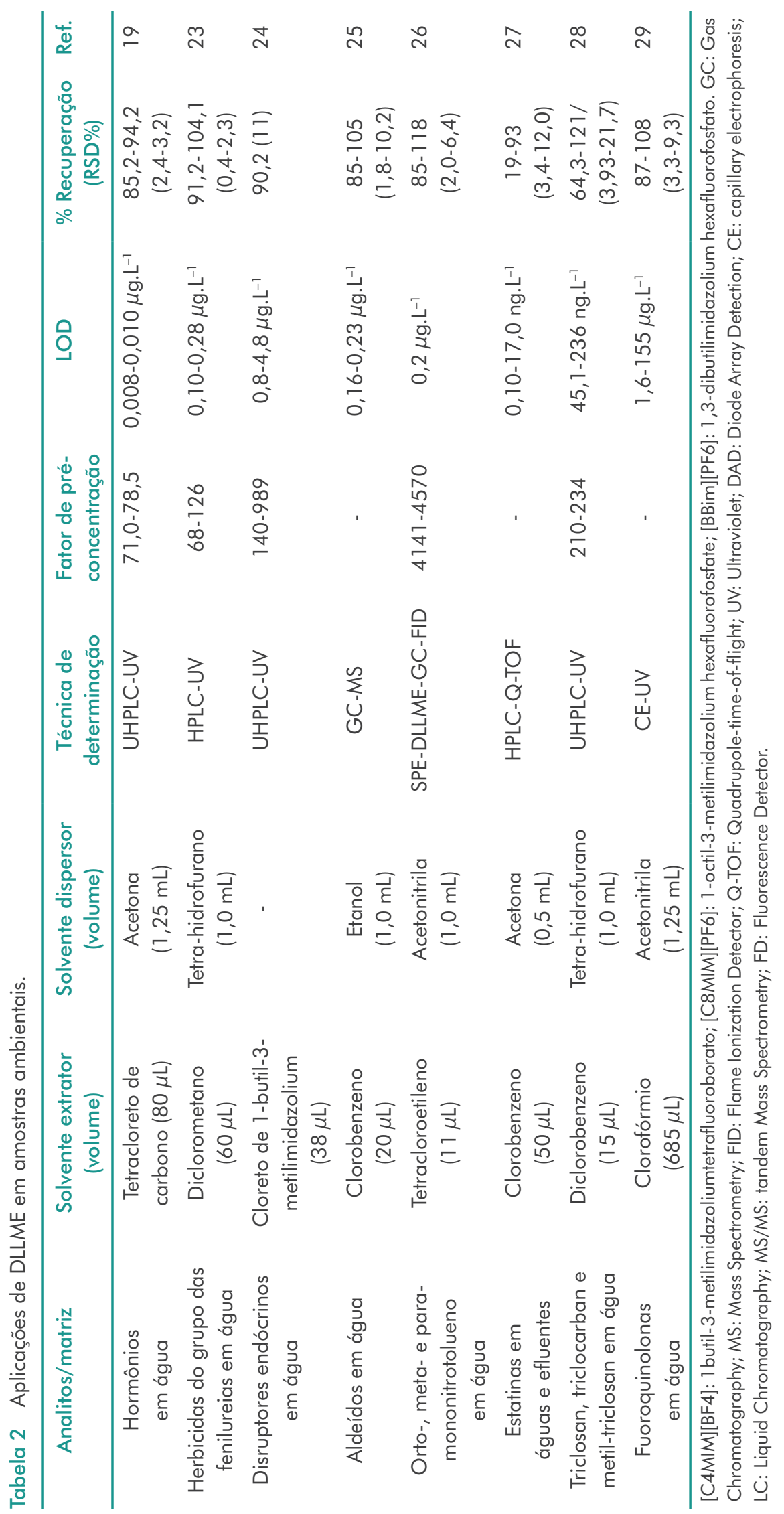




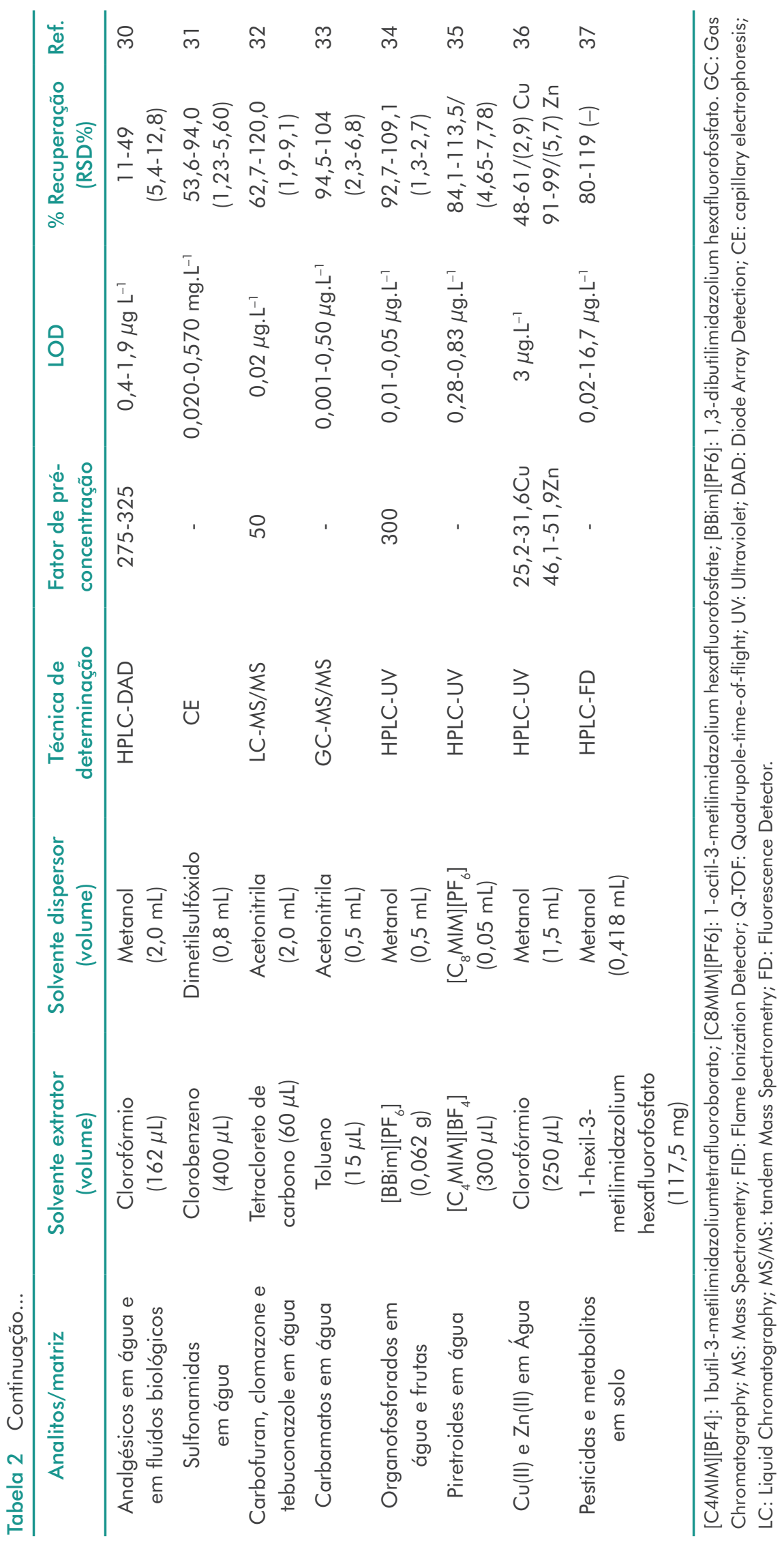




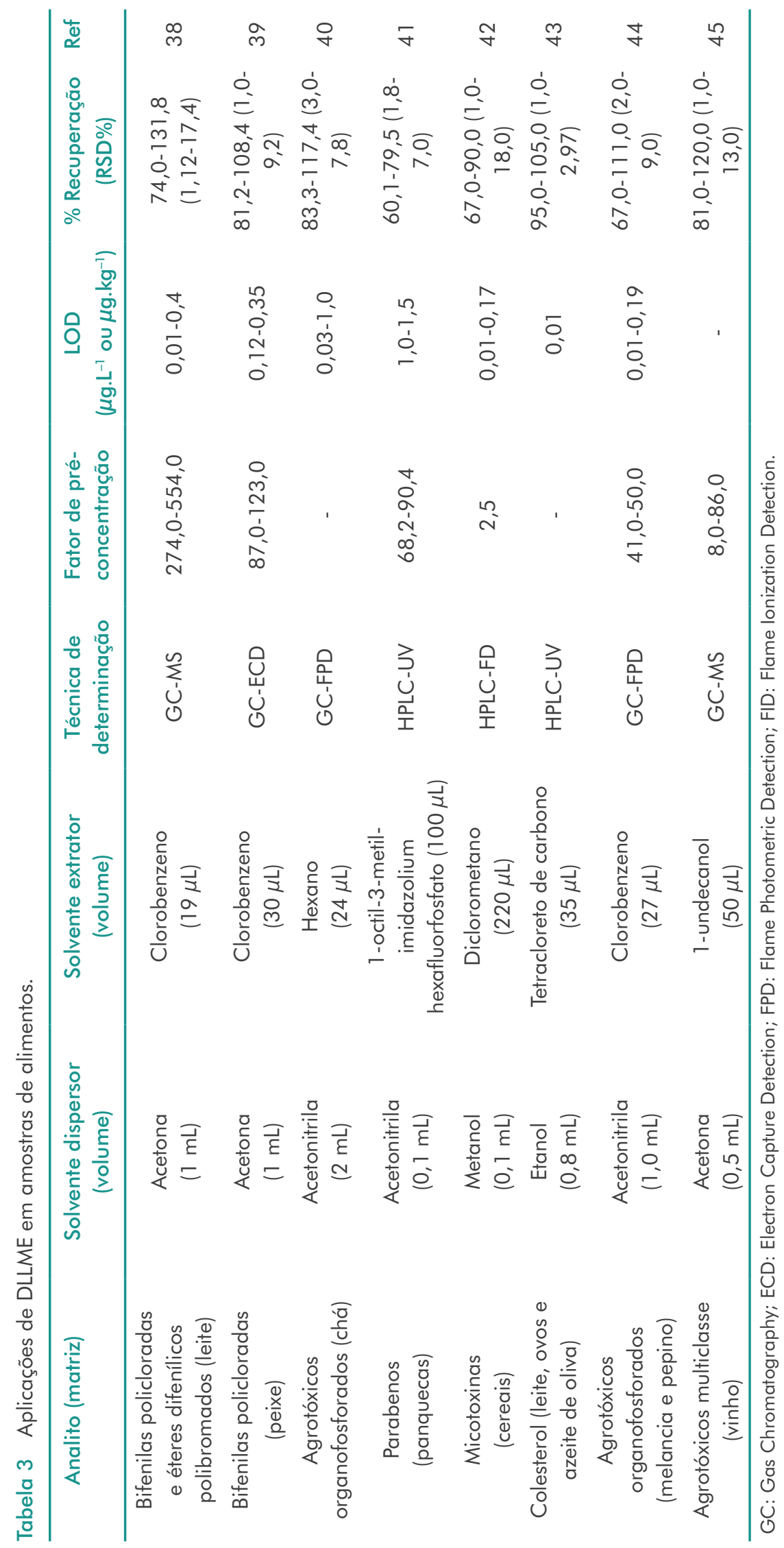




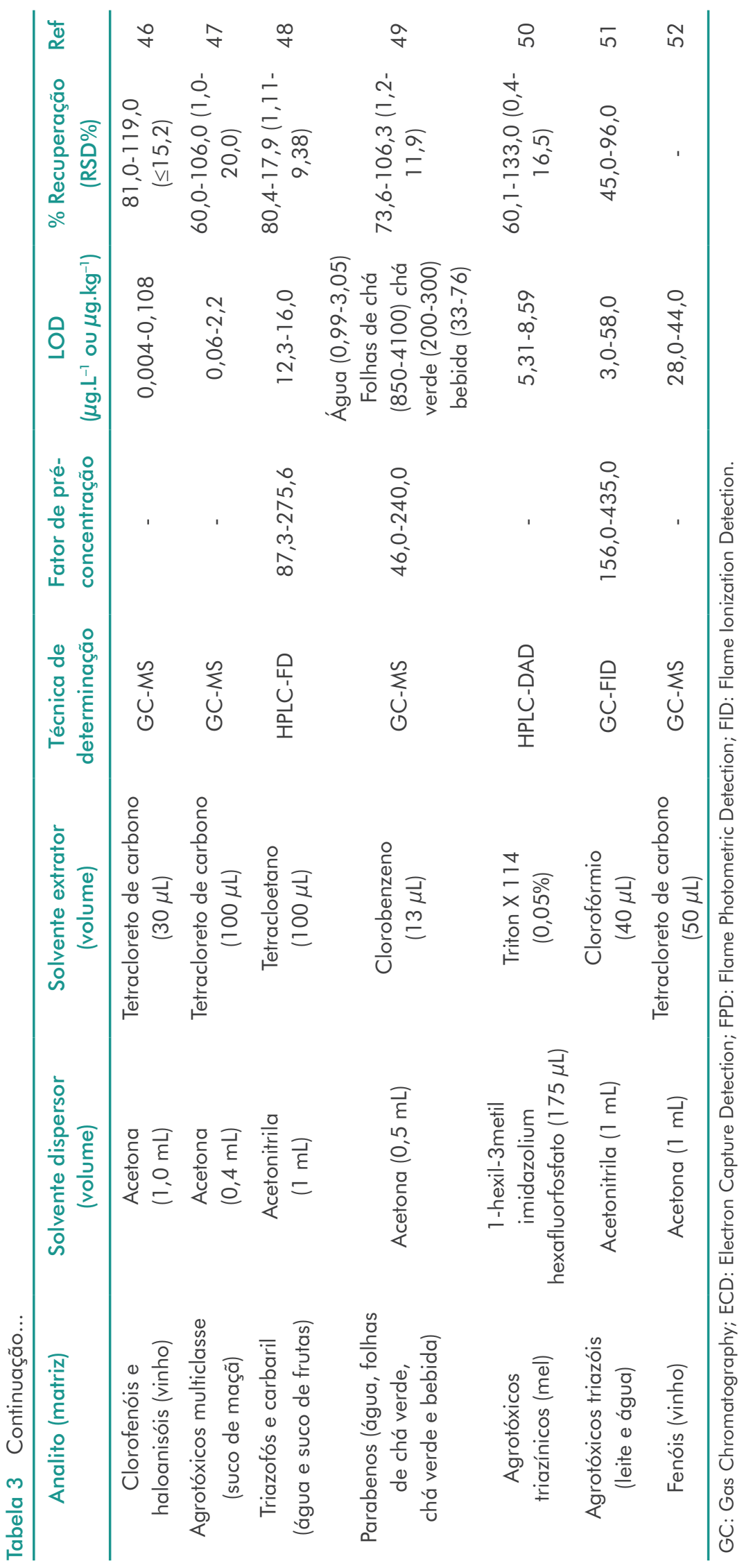


ventes é criticado devido a sua toxicidade. No entanto, a quantidade de solventes usados em DLLME é muito reduzida. Embora a DLLME tenha sido proposta para solventes mais densos do que a água, Rodriguéz-Cabo et al. ${ }^{[4]}$ desenvolveram uma nova proposta para a determinação de resíduos de fungicidas em vinho, empregando $50 \mu \mathrm{L}$ de 1-undecanol como solvente de extração. Este solvente caracteriza-se por ser não clorado e de menor densidade que a água. Os 10 analitos de interesse foram analisados por GC-MS (do inglês Gas Chromatography coupled to Mass Spectrometry). O método apresentou RSD\% inferiores a $13 \%$ e os valores de LOQ variaram entre 0,2 e $3,2 \mu \mathrm{g} . \mathrm{L}^{-1}$. Os valores de recuperação obtidos estão na faixa de 81 e 120\%, com fatores de pré-concentração entre 8 e 86 vezes.

Os excelentes resultados obtidos com a utilização da técnica de DLLME também podem ser observados quando esta é combinada a outras técnicas de preparo de amostras. Yan et al. ${ }^{[53]}$ empregaram DLLME combinada com a técnica de Dispersão da Matriz em Fase Sólida (MSPD, do inglês Matrix Solid Phase Dispersion) para a determinação de corantes do tipo Sudão em amostras de gema de ovo por HPLC-UV (do inglês High Performance Liquid Chromatography with Ultraviolet Detection). Durante o procedimento de MSPD a amostra foi diretamente misturada com um polímero de impressão molecular (MIP, do inglês Molecularly Imprinted Polymer) formado por anilina e naftol. Uma mistura de acetona:ácido acético $(95: 5, \mathrm{v} / \mathrm{v})$ foi empregada como solvente de eluição na MSPD e, após, como solvente de dispersão na DLLME. O método apresentou boa linearidade, suas recuperações em três níveis de fortificação variaram entre 87,2 e $103,5 \%$, com RSD $\% \leq 6,1$. Os valores de LODs do método variaram entre $2,9 \mathrm{e}$ 6,7 $\mu \mathrm{g} \cdot \mathrm{kg}^{-1}$, demonstrando o bom desempenho da combinação entre as técnicas de MIPs-MSPD e DLLME.

O uso de ultrassom para facilitar a extração também tem sido empregado. Os estudos relatam que o uso do ultrassom aumenta a eficiência de extração, uma vez que microgotas menores são produzidas quando a vibração ultrassônica é empregada. A UA-DLLME (ultrasound-assisted-DLLME) foi utilizada no trabalho desenvolvido por Chen et al. ${ }^{[54]}$ que descrevem um método simples e com baixo consumo de solventes para a determinação de fulerenos em amostras de água. Os compostos foram determinados por LC-APPI-MS/MS. O método consistiu na injeção de $1 \mathrm{~mL}$ de 2-propanol (dispersor) e $10 \mu \mathrm{L}$ de brometo de benzila (extrator) em $10 \mathrm{~mL}$ da amostra $\mathrm{pH} 10$, contendo $1 \%$ de $\mathrm{NaCl}$. As amostras foram submetidas ao ultrassom por 1 minuto, depois foram centrifugadas e $5 \mu \mathrm{L}$ da fase sedimentada foi injetado. O método apresentou valores de LOQ entre 8 e 150 ng. $\mathrm{L}^{-1}$, com precisão menor que $12 \%$ e exatidão entre 70 e $86 \%$.

Apesar das importantes vantagens da técnica DLLME anteriormente citadas, a dificuldade de automação, devido à necessidade de etapas de separação de fases e centrifugação, é considerada uma desvantagem. A fim de minimizar este problema, Andruch et al. ${ }^{[55]}$ propuseram a combinação entre um sistema de injeção sequencial de análise (SIA, do inglês Sequencial Injection Analysis) e a técnica de DLLME para a determinação de tiocianato em amostra de saliva empregando detector espectrofotométrico. Os resultados obtidos demonstram a eficiência do sistema proposto, sendo que a combinação SIA-DLLME apresentou LOD de 17,0 $\mu$ g.L $\mathrm{L}^{-1}$, e o método convencional por DLLME apresentou LOD de 100,0 $\mu \mathrm{g} . \mathrm{L}^{-1}$. Além disso, outra grande vantagem desta combinação foi o tempo de 3 segundos para a realização da etapa de extração por DLLME.

A completa automação da técnica foi proposta por Maya et al. ${ }^{[56]}$, empregando injeção em fluxo com multisseringas (MSFIA, Multisyringe Flow Injection Analysis) e solventes extratores menos densos que a água. Quando hifenado com 
a Cromatografia Líquida, o método é compacto, simples, fácil de operar e rápido. A aplicação foi demonstrada para extração de benzopireno, utilizando 1-octanol (extrator) e acetonitrila (dispersor) na proporção $(7,5 / 92,5, \mathrm{v} / \mathrm{v})$.

\section{Conclusões e perspectivas}

Apesar de ser uma técnica de preparo de amostra relativamente nova, muitas modificações têm sido introduzidas desde sua proposição em 2006. Inicialmente foi utilizada para preparo de amostras para GC e posteriormente para LC, FAAS (do inglês Flame Atomic Absorption Spectrometry), GF-AAS (do inglês Graphite Furnace Atomic Absorption Spectrometry). As modificações em relação ao procedimento original incluem a utilização de líquidos iônicos e de solvente extratores com densidade menor que a da água. Algumas publicações relatam o desenvolvimento de DLLME com derivação e/ou complexação simultânea e extração de íons metálicos com o auxílio de agentes quelantes ${ }^{[57]}$.

A DLLME tem sido utilizada com maior frequência para extração e pré-concentração de agrotóxicos em amostras aquosas. Também tem sido utilizada para extração de analitos orgânicos, como produtos farmacêuticos, aminas, fenóis e outros em amostras aquosas e alimentos ${ }^{[58]}$.

A DLLME quando combinada a outras técnicas visando a pré-concentração dos analitos de interesse é uma alternativa à etapa de evaporação do extrato. Desta forma, diminui a variação da eficiência do processo de extração, principal fonte de incerteza do preparo de amostras.

Em conjunto com outras técnicas de preparo de amostra, a DLLME pode ser utilizada para a extração de analitos de amostras sólidas. Algumas publicações relatam este tipo de aplicação, na qual os analitos são extraídos da matriz, utilizando a extração sólido-líquido, empre- gando o método QuEChERS, ou, no caso de metais, a mineralização ${ }^{[36]}$. Neste caso, a DLLME é utilizada para concentrar os extratos. Outra tendência que pode ser observada em publicações recentes é o uso da DLLME em conjunto com a SPE (do inglês Solid Phase Extraction), com a extração por fluído supercrítico e com a SBSE (do inglês Stir Bar Sorptive Extraction). Estas modificações permitem a obtenção de maior seletividade e menores valores de $\mathrm{LOD}^{[58]}$.

Uma tendência de uso da DLLME, ainda com poucas aplicações descritas na literatura, é a utilização de sorventes em suspensão em amostras submetidas à DLLME. Exemplos deste tipo de aplicação são o emprego de $\operatorname{MIPs}^{[3,59]}$, que são posteriormente removidos da amostra por filtração, e o emprego de nanopartículas magnéticas quimicamente modificadas ${ }^{[60]}$, que são removidas por um campo magnético. Conforme sugerido por Grzeskowiak e Grzeskowiak. ${ }^{[57]}$, a combinação destas duas ideias, ou seja, nanopartículas de MIP com núcleos magnéticos, pode trazer alta seletividade e facilidade de uso à DLLME com utilização de sorventes sólidos em suspensão.

A automação da DLLME deve ser uma tendência no futuro, atualmente poucos artigos relatam o desenvolvimento de um procedimento analítico utilizando DLLME on-line ${ }^{[55,56]}$.

As considerações descritas neste artigo sugerem uma perspectiva promissora nas aplicações da DLLME. A versatilidade desta técnica irá permitir sua aplicação para a extração de diferentes tipos de analitos nas mais variadas amostras.

\section{Agradecimentos}

Os autores agradecem ao Conselho Nacional de Desenvolvimento Científico e Tecnológico (CNPq) e à Coordenação de Aperfeiçoamento de Pessoal de Nível Superior (CAPES) o apoio financeiro e bolsas concedidas. 


\section{Referências}

1 OjedaCB, RojasFS.Separation and Preconcentration by Dispersive Liquid-Liquid Microextraction Procedure: Recent Applications. Chromatographia 2011; 74:651679. http://dx.doi.org/10.1007/s10337-011-2124-1

2 Rezaee M, Assadi Y, Hossein MM, Aghaee E, Ahmadia F, Berijani S. Determination of organic compounds in water using dispersive liquid-liquid microextraction. Journal Chromatography A 2006; 1116:1-9. http:// dx.doi.org/10.1016/j.chroma.2006.03.007

3 Rezaee M, Yamini Y, Faraji M. Evolution of dispersive liquid-liquid microextraction method. Journal of Chromatography A 2010; 1217:2342-2357. http:// dx.doi.org/10.1016/j.chroma.2009.11.088

4 Yazdi AS, Amiri A. Liquid-phase microextraction. Trends in Analytical Chemistry 2010; 29(1):1-14. http://dx.doi.org/10.1016/j.trac.2009.10.003

5 Berijani S, Assadi Y, Anbia M, Hosseini MRM, Aghaee E. Dispersive liquid-liquid microextraction combined with gas chromatography-flame photometric detection Very simple, rapid and sensitive method for the determination of organophosphorus pesticides in water. Journal of Chromatography A 2006; 1123:1-9. http://dx.doi.org/10.1016/j.chroma.2006.05.010

6 Oliveira ARM, Magalhães IRS, Santana FJM, Bonato PS. Microextração em Fase Líquida (LPME): Fundamentos da técnica e aplicações na análise de fármacos em fluidos biológicos. Química Nova 2008; 31(3):637-644. http://dx.doi.org/10.1590/ S0100-4042200800030003

7 Harris DC. Análise Química Quantitativa. 8th ed. Rio de Janeiro: LTC; 2012. 886 p.

8 Bjergaard SP, Rasmussen KE. Liquid-phase microextraction with porous hollow fibers, a miniaturized and highly flexible format for liquid-liquid extraction. Journal Chromatography A 2008; 1184:132-142. http://dx.doi.org/10.1016/j. chroma.2007.08.088

9 Ho TS, Vasskog T, Anderssen T, Jensen E, Rasmussen KE, Bjergaard SP. 25,000-fold pre-concentration in a single step with liquid-phase microextraction. Analytica Chimica Acta 2007; 592:1-8. http://dx.doi. org/10.1016/j.aca.2007.04.014

10 Kozani RR, Assadi Y, Shemirani F, Hosseini MRM, Jamali MR. Part-per-trillion determination of chlorobenzenes in water using dispersive liquid-liquid microextraction combined gaschromatography- electron capture detection. Talanta 2007; 72:387-393. http://dx.doi.org/10.1016/j.talanta.2006.10.039

11 Herrera-Herrera AV, Asensio-Ramos M, HernandezBorges J, Rodriguez-Delgado MA. Dispersive liquid-liquid microextraction for determination of organic analytes. Trends in Analytical Chemistry 2010; 29(7):728-751. http://dx.doi. org/10.1016/j.trac.2010.03.016

12 Zang XH, Wu QH, Zhang MY, Xi GH, Wang S. Developments of Dispersive Liquid-Liquid Microextraction Technique. Chinese Journal of Analytical Chemistry 2009; 37(2):161-168. http:// dx.doi.org/10.1016/S1872-2040(08)60082-1

13 Merck Chemicals. Solventes para Cromatografia Líquida. Lichrosolv. [cited feb 2012]. Available from: http://www.merck-chemicals.com/brazil/ copy-of-solventes-para-cromatografia-liquidalichrosolv-/c_3xab.s1OK68AAAEuBEdQn6U9.

14 Anthemidis AN, Ioannou KIG. Recent developments in homogeneous and dispersive liquid-liquid extraction for inorganic elements determination. A review. Talanta 2009; 80:413-421. http://dx.doi. org/10.1016/j.talanta.2009.09.005

15 Liu L, Cheng J, Matsadiq G, Zhou H, Li J. Application of DLLME to the Determination of Pyrethroids in Aqueous Samples. Chromatographia 2010; 72:1017$1020 . \quad$ http://dx.doi.org/10.1365/ s10337-010-1732-5 0009-5893/10/11

16 Leong $M$, Huang S. Dispersive liquid-liquid microextraction method based on solidification of floating organic drop combined with gas chromatography with electron-capture or mass spectrometry detection. Journal of Chromatography A 2008; 1211:8-12. http://dx.doi.org/10.1016/j. chroma.2008.09.111

17 Leong MI, Chang CC, Fuh MR, Huang SD. Low toxic dispersive liquid-liquid microextraction using halosolvents for extraction of polycyclic aromatic hydrocarbons in water samples. Journal of Chromatography A 2010; 1217:5455-5461. http:// dx.doi.org/10.1016/j.chroma.2010.06.056

18 Krylov VA, Krylov AV, Mosyagin PV, Matkivskaya YO. Liquid-Phase Microextraction Preconcentration of Impurities. Journal of Analytical Chemistry 2011; 66(4):331-350. http://dx.doi. org/10.1134/S1061934811040101

19 Hadjmohammadi MR, Ghoreishi SS. Determination of Estrogens in Water Samples Using Dispersive 
Liquid Liquid Microextraction and High Performance Liquid Chromatography. Acta Chimica Slovenica 2011; 58:765-771.

20 Nagaraju D, Huang SD. Determination of triazine herbicides in aqueous samples by dispersive liquidliquid microextraction with gas chromatography-ion trap mass spectrometry. Journal of Chromatography A 2007; 1161:89-97. http://dx.doi.org/10.1016/j. chroma.2007.05.065

21 Caldas SS, Gonçalves FF, Primel EG, Prestes OD, Martins ML, Zanella R. Principais técnicas de preparo de amostra para a determinação de resíduos de agrotóxicos em água por cromatografia líquida com detecção por arranjo de diodos e por espectrometria de massas. Química Nova 2011; 34(9):1604-1617. http://dx.doi.org/10.1590/S0100-40422011000900021

22 Farhadi K, Matin AA, Hashemi P. LC Determination of Trace Amounts of Phenoxyacetic Acid Herbicides in Water after Dispersive Liquid-Liquid Microextraction. Chromatographia 2009; 69:45-49. http://dx.doi. org/10.1365/s10337-008-0815-z0009-5893/09/01

23 Chou T, Lin S, Fuh M. Determination of phenylurea herbicides in aqueous samples using partitioned dispersive liquid-liquid microextraction. Talanta 2009; 80:493-498. http://dx.doi.org/10.1016/j. talanta.2009.07.005

24 López-Darias J, Pino V, Ayala JH, Afonso AM. In-situ ionic liquid-dispersive liquid-liquid microextraction method to determine endocrine disrupting phenols in seawaters and industrial effluents. Microchimica Acta 2011; 174:213-222. http://dx.doi.org/10.1007/ s00604-011-0636-X

25 Ye Q, Zheng D, Liu L, Hong L. Rapid analysis of aldehydes by simultaneous microextraction and derivatization followed by GC-MS. Journal of Separation Science 2011; 34:1607-1612. http://dx.doi. org/10.1002/jssc.201100145

26 Sobhi HR, Farahani H, Kashtiaray A, Farahani MR. Tandem use of solid-phase extraction and dispersive liquid-liquid microextraction for the determination of mononitrotoluenes in aquatic environment. Journal of Separation Science 2011; 34:1035-1040. http://dx.doi. org/10.1002/jssc.201000529

27 Martín J, Buchberger W, Alonso E, Himmelsbach M, Aparicio I. Comparison of different extraction methods for the determination of statin drugs in wastewater and river water by HPLC/Q-TOF-MS. Talanta 2011; 85:607-615. http://dx.doi.org/10.1016/j. talanta.2011.04.017
28 Guo J, LiX, Cao X, Li Y, Wang X, XuX. Determination of triclosan, triclocarban and methyl-triclosan in aqueous samples by dispersive liquid-liquid microextraction combined with rapid liquid chromatography. Journal of Chromatography A 2009; 1216:3038-3043. http:// dx.doi.org/10.1016/j.chroma.2009.02.021

29 Herrera-Herrera AV, Hernandez-Borges J, BorgesMiquel T, Rodriguez-Delgado MA. Dispersive liquid-liquid microextraction combined with nonaqueous capillary electrophoresis for the determination of fluoroquinolone antibiotics in Waters. Electrophoresis 2010; 31:3457-3465. http:// dx.doi.org/10.1002/elps.201000285

30 Saraji M., Boroujeni MK, Bidgoli AAH. Comparison of dispersive liquid-liquid microextraction and hollow fiber liquid-liquid-liquid microextraction for the determination of fentanyl, alfentanil, and sufentanil in water and biological fluids by highperformance liquid chromatography. Analytical and Bioanalytical Chemistry 2011; 400:2149-2158. http:// dx.doi.org/10.1007/s00216-011-4874-x

31 Wen Y, Li J, Zhang W, Chen L. Dispersive liquidliquid microextraction coupled with capillary electrophoresis for simultaneous determination of sulfonamides with the aid of experimental design. Electrophoresis 2011; 32:2131-2138. http://dx.doi. org/10.1002/elps.201100142

32 Caldas SS, Costa FP, Primel EG. Validation of method for determination of different classes of pesticides in aqueous samples by dispersive liquid liquid microextraction with liquid chromatography tandem mass spectrometric detection. Analytica Chimica Acta 2010; 32:55-62 http://dx.doi.org/10.1016/j. aca.2010.03.004

33 Chen H, Chen R, Li S. Low-density extraction solventbased solvent terminated dispersive liquid-liquid microextraction combined with gas chromatographytandem mass spectrometry for the determination of carbamate pesticides in water samples. Journal of Chromatography A 2010; 1217:1244-1248. http:// dx.doi.org/10.1016/j.chroma.2009.12.062

34 HeL;LuoX;JiangX,QuL.Anew1,3-dibutylimidazolium hexafluorophosphate ionic liquid-based dispersive liquid-liquid microextraction to determine organophosphorus pesticides in water and fruit samples by high-performance liquid chromatography. Journal of Chromatography A 2010; 1217:5013-5020. http://dx.doi.org/10.1016/j.chroma.2010.05.057

35 Zhao R, Wang X, Li F, Wang S, Zhang L, C Cheng. Ionic liquid/ionic liquid dispersive liquid- 
liquid microextraction. Journal of Separation Science 2011; 34:830-836. http://dx.doi.org/10.1002/ jssc. 201000802

36 Farajzadeh MA, Bahram M, Vardast MR. Central Composite Design Applied to Optimization of Dispersive Liquid-Liquid Microextraction of $\mathrm{Cu}(\mathrm{II})$ and $\mathrm{Zn}(\mathrm{II})$ in Water Followed by High Performance Liquid Chromatography Determination. CLEAN - Soil, Air, Water 2010; 38(5-6):466-477. http://dx.doi.org/10.1002/clen.200900257

37 Asensio-Ramos M, Hernandez-Borges J, BorgesMiquel TM, Rodriguez-Delgado MA. Ionic liquid-dispersive liquid-liquid microextraction for the simultaneous determination of pesticides and metabolites in soils using high-performance liquid chromatography and fluorescence detection. Journal of Chromatography A 2011; 1218:4808-4816. http:// dx.doi.org/10.1016/j.chroma.2010.11.030

38 Liu X, Zhao A, Zhang A, Liu H, Xiao W, Wang C et al. Dispersive liquid-liquid microextraction and gas chromatography-mass spectrometry determination of polychlorinated biphenyls and polybrominated diphenyl ethers in milk. Journal of Separation Science 2011; 34:1084-1090. http://dx.doi.org/10.1002/ jssc. 201000767

39 Hu Y, Li Y, Zhang W, Wang H, Huang C, Zhang M et al. Dispersive liquid-liquid microextraction followed by gas chromatography-electron capture detection for determination of polychlorinated biphenyls in fish. Journal of Separation Science 2009; 32:2103-2108. http://dx.doi.org/10.1002/jssc.200900027

40 Moinfar S, Hosseini MRM. Development of dispersive liquid-liquid microextraction method for the analysis of organophosphorus pesticides in tea. Journal of Hazardous Materials 2009; 169:907-911. http://dx.doi. org/10.1016/j.jhazmat.2009.04.030

41 Yang P, Ren H, Qiu H, Liu X, Jiang S. Determination of four trace preservatives in street food by ionic liquidbased dispersive liquid-liquid micro-extraction. Chemical Papers 2011; 65(6):747-753. http://dx.doi. org/10.2478/s11696-011-0071-9

42 Fu L, Liu X, Hu J, Zhao X, Wang H, Wang X. Application of dispersive liquid-liquid microextraction for the analysis of triazophos and carbaryl pesticides in water and fruit juice samples. Analitica Chemica Acta 2009; 632:289-295. http://dx.doi.org/10.1016/j. aca.2008.11.020

43 Daneshfar A, Khezeli T, Lotfi HJ. Determination of cholesterol in food samples using dispersive liquid- liquid microextraction followed by HPLC-UV. Journal of Chromatography B 2009; 877:456-460. http://dx.doi. org/10.1016/j.jchromb.2008.12.050

44 Zhao E, Zhao W, Han L, Jiang S, Zhou Z. Application of dispersive liquid-liquid microextraction for the analysis of organophosphorus pesticides in watermelon and cucumber. Journal of Chromatography A 2007; 1175:137-140. http://dx.doi.org/10.1016/j. chroma.2007.10.069

45 Cabo TR, Rodríguez I, Ramil M, Cela R. Dispersive liquid-liquid microextraction using non-chlorinated, lighter than water solvents for gas chromatography-mass spectrometry determination of fungicides in wine. Journal of Chromatography A 2011; 1218:6603-6611. http://dx.doi.org/10.1016/j. chroma.2011.07.054

46 Campillo N, Viñas P, Cacho JI, Peñalver R, Córdoba $\mathrm{MH}$. Evaluation of dispersive liquidliquid microextraction for the simultaneous determination of chlorophenols and haloanisoles in wines and cork stoppers using gas chromatographymass spectrometry. Journal of Chromatography A 2010; 1217:7323-7330. http://dx.doi.org/10.1016/j. chroma.2010.09.058

47 Cunha SC, Fernandes JO, Oliveira MBPP. Fast analysis of multiple pesticide residues in apple juice using dispersive liquid-liquid microextraction and multidimensional gas chromatographymass spectrometry. Journal of Chromatography A 2009; 1216:8835-8844. http://dx.doi.org/10.1016/j. chroma.2009.10.051

48 Fu L, Liu X, Hu J, Zhao X, Wang H, Wang X. Application of dispersive liquid-liquid microextraction for the analysis of triazophos and carbaryl pesticides in water and fruit juice samples Analityca Chimica Acta 2009; 632:289-295. http://dx.doi.org/10.1016/j. aca.2008.11.020

49 Han Y, Jia X, Liu X, Duan T, Chen H. DLlME Combined with GC-MS for the Determination of Methylparaben, Ethylparaben, Propylparaben and Butylparaben in Beverage Samples. Chromatographia 2010; 72:351-355. http://dx.doi. org/10.1365/s10337-010-1646-2 0009-5893/10/08

50 Wang Y, You J, Ren R, Xiao Y, Gao S, Zhang H et al. Determination of triazines in honey by dispersive liquid-liquid microextraction high-performance liquid chromatography. Journal of Chromatography A 2010; 1217:4241-4246. http://dx.doi.org/10.1016/j. chroma.2010.03.031 
51 Farajzadeh MA, Djozan D, Mogaddan MRA. Extraction and preconcentration technique for triazole pesticides from cow milk using dispersive liquid-liquid microextraction followed by GC-FID and GC-MS determinations. Journal of Separations Science 2011;34:1309-1316. http://dx.doi.org/10.1002/ jssc. 201000928

52 Fariña L, Boido E, Carrau F, Delacassa E. Determination of volatile phenols in red wines by dispersive liquidliquid microextraction and gas chromatography-mass spectrometry detection. Journal of Chromatography A 2007;1157:46-50. http://dx.doi.org/10.1016/j. chroma.2007.05.006

53 Yan H, Wang H, Qiao J, Yang G. Molecularly imprinted matrix solid-phase dispersion combined with dispersive liquid-liquid microextraction for the determination of four Sudan dyes in egg yolk. Journal of Chromatography A 2011; 1218:2182-2188 http:// dx.doi.org/10.1016/j.chroma.2011.02.042

54 Chen $\mathrm{H}$, Ding $\mathrm{W}$, Determination of aqueous fullerene aggregates in water by ultrasoundassisted dispersive liquid-liquid microextraction with liquid chromatography-atmospheric pressure photoionization-tandem mass spectrometry. Journal of Chromatography A 2012; 1223:15-23. http://dx.doi. org/10.1016/j.chroma.2011.12.034

55 Andruch V, Acebal CC, Jkrlikova J, Sklenatova H, Solich P, Balogh IS et al. Automated on-line dispersive liquid-liquid microextraction based on a sequential injection system. Microchemical Journal 2012; 100:7782. http://dx.doi.org/10.1016/j.microc.2011.09.006

56 Maya F, Estela JM, Cerdà V. Completely automated in-syringe dispersive liquid-liquid microextraction using solvents lighter than water. Analytical and Bioanalytical Chemistry 2012; 402:1383-1388. http:// dx.doi.org/10.1007/s00216-011-5572-4

57 Grzeskowiak AZ, Grzeskowiak T. Dispersive liquid-liquid microextraction. Trends in Analytical Chemistry 2011; 30(9):1382-1399. http://dx.doi. org/10.1016/j.trac.2011.04.014

58 Asensio-Ramos M, Revelo-Perez LM, GonzálezCurbelo MA, Hernandez-Borges J. Liquid phase microextraction applications in food analysis. Journal of Chromatography A 2011; 1218:7415-7437. http:// dx.doi.org/10.1016/j.chroma.2011.05.096

59 Ebrahimzadeh H, Abedi H, Yamini Y, Adlnasab L. Molecular-imprinted polymer extraction combined with dispersive liquid-liquid micro-extractionfor ultra-preconcentration of mononitrotoluene. Journal of Separation Science 2010; 33:3759-3766. http:// dx.doi.org/10.1002/jssc.201000376

60 Luo YB, Shi ZG, Gao Q, Fenga YQ. Magnetic retrieval of graphene: Extraction of sulfonamide antibiotics from environmental water samples. Journal of Chromatography A 2011; 1218:1353-1358. http:// dx.doi.org/10.1016/j.chroma.2011.01.022 